\title{
La Confédération et la FMH arrivent au même résultat
}

\section{Esther Kraft}

Cheffe de la division DDQ

Près de 7000 cabinets médicaux et centres de soins ambulatoires ont pris part au relevé «MAS 2015» (pour Medical Ambulatory Structure) et livré des informations concernant leur activité, chiffre d'affaires et résultat d'exploitation. Le taux de participation s'est élevé à environ $75 \%$. Le rapprochement entre les chiffres obtenus par l'étude MAS 2015 et les données collectées par les médecins montre des résultats comparables malgré des sources de données différentes. Le résultat moyen des activités au cabinet et des autres activités se monte à 155000 francs, et $89 \%$ de l'activité au cabinet provient des primes.

\section{Contexte}

Le 20 mars 2018, l'Office fédéral de la statistique (OFS) a publié l'analyse de la participation et de la population du premier relevé des données structurelles des cabinets médicaux et centres ambulatoires (MAS) [1]. Environ trois quarts des cabinets médicaux et centres de soins ambulatoires sollicités ont fourni des informations concernant leur situation. Près de 7000 cabinets médicaux et centres ambulatoires (soit la moitié des entreprises appartenant à la population pondérée) ont rempli le questionnaire et livré des données. Sur la base de ces éléments, l'OFS a été en mesure de publier, le 10 avril 2018, les premiers résultats du relevé MAS 2015 [2], effectué à des fins statistiques en conformité avec l'article 23 de la loi fédérale sur l'assurance-mala-
Tableau 1: Nombre de médecins en pratique ambulatoire en 2015 (cabinets, centres de soins ambulatoires).

\begin{tabular}{lllc}
\hline & MAS 2015 & $\begin{array}{l}\text { Statistique médicale de la FMH } \\
\text { (secteur ambulatoire) 2015 }\end{array}$ & Delta \\
\hline $\begin{array}{l}\text { Médecins en pratique } \\
\text { ambulatoire }\end{array}$ & 17599 & 18128 & $3,0 \%$ \\
\hline Equivalents temps plein & 14044 & 15046 & $7,1 \%$ \\
\hline $\begin{array}{l}\text { Taux d'activité } \\
\text { (demi-jour par semaine) }\end{array}$ & 7,6 & 8,3 & $9,2 \%$ \\
\hline $\begin{array}{l}\text { Taux, hommes } \\
\text { (demi-jour par semaine) }\end{array}$ & 8,1 & 8,9 & $9,9 \%$ \\
\hline $\begin{array}{l}\text { Taux, femmes } \\
\text { (demi-jour par semaine) }\end{array}$ & 7,6 & 6,9 & $-9,2 \%$ \\
\hline
\end{tabular}

die (LAMal) et en application de la loi sur la statistique fédérale (LSF).

Le premier relevé peut être considéré comme un succès. L'OFS a même précisé que le «fait que la FMH, la plus importante association professionnelle, ainsi que ses partenaires (Caisse des médecins et NewIndex) aient soutenu la livraison des données à usage statistique, a été l'un des prérequis pour garantir une participation du corps médical au relevé MAS 2015. Les interfaces avec les bases de données existantes, appartenant respectivement à la Caisse des médecins (RoKo), NewIndex, la FMH et MedReg, ont facilité la saisie des données pour les entreprises interrogées: d'une part, elles ont permis aux entreprises d'importer en quelques clics des données existantes, et d'autre part, de garantir la bonne qualité des données livrées» [1].

\section{Analyse des données par la FMH}

Les résultats du relevé MAS 2015 revêtent beaucoup d'importance et d'intérêt pour la FMH et le corps médical. En se fondant sur la loi sur la statistique, l'ordonnance concernant l'exécution des relevés statistiques fédéraux et la loi sur la protection des données, la FMH a recouru à la possibilité d'exiger à l'OFS une formulation claire des objectifs concernant la recherche, la planification et les statistiques en lien avec les données du relevé MAS 2015.

La comparaison des résultats de ce relevé avec les données des médecins du point de vue de la qualité, de la 
Tableau 2: Dépenses et chiffres d'affaires des entreprises individuelles, MAS 2015 et RoKo.

\begin{tabular}{|c|c|c|c|}
\hline & MAS 2015 & RoKo & Delta \\
\hline Chiffre d'affaires & 544806 & 556980 & $2,2 \%$ \\
\hline de l'activité au cabinet & $93 \%$ & $92 \%$ & \\
\hline Autres affaires & $1 \%$ & $1 \%$ & \\
\hline Dépenses matérielles, activité méd. & $29 \%$ & & \\
\hline Assurance & $15 \%$ & $14 \%$ & \\
\hline Autres dépenses & $23 \%$ & & \\
\hline Résultats d'exploitation & 154414 & 167559 & $8,1 \%$ \\
\hline
\end{tabular}

pertinence et de l'interprétation vise à permettre une discussion transparente sur la base de données réelles et validées.

\section{Comparaison des chiffres des médecins et des données financières}

Une première phase de l'analyse s'est penchée sur la comparaison du nombre de médecins en exercice et de leur taux d'activité selon les chiffres du relevé MAS 2015 et ceux de la statistique médicale de la FMH. Une deuxième phase a mis en perspective les données financières du relevé MAS avec celles rendues disponibles par l'étude permanente sur les coûts (RoKo).

Dans sa publication pour l'année 2015, l'OFS dénombre 17599 médecins en pratique ambulatoire, soit 14044 équivalents temps plein. Pour la même année, la statistique médicale de la FMH fait, quant à elle, état de 18858 médecins en pratique ambulatoire, soit 15046 équivalents temps plein (cf. tab. 1).

Avec les données financières de 2015, l'OFS arrive à un chiffre d'affaires global moyen de 545000 francs pour les cabinets médicaux constitués en entreprise individuelle. Les dépenses globales de ces cabinets s'élèvent en moyenne à 390000 francs équivalant à un taux de coût (dépenses / chiffre d'affaires) de $72 \%$ en moyenne. Par conséquent, le résultat d'exploitation des cabinets particuliers s'est élevé à 155000 francs en 2015. En comparaison, les données de l'étude permanente des coûts (RoKo) présentent un taux de coût de $70 \%$ et un résultat d'exploitation de 167000 francs environ.

FMH

Division DDQ

Elfenstrasse 18

CH-3000 Berne 15

Tél. 031359111

ddq[at]fmh.ch une entreprise individuelle ne sont pas considérés comme des salariés et, par conséquent, leur revenu n'apparaît pas dans les frais de personnel.

D’après les données MAS 2015, le chiffre d'affaires est généré à 89\% par l'assurance-maladie obligatoire. Par conséquent, avec un un résultat d'exploitation moyen de 155000 francs, le propriétaire d'un cabinet gagne en moyenne 137950 francs issus directement des primes de l'AOS.

\section{Conclusion}

Il est réjouissant de voir que les relevés réalisés indépendamment par la Confédération et le corps médical présentent des résultats comparables concernant le nombre de médecins en exercice et leur taux d'activité ainsi que les données financières. Les données obtenues offrent ainsi une bonne base en vue d'une discussion transparente et réaliste sur le nombre de médecins et les conditions-cadres financières des médecins installés.

Nous saisissons ici l'occasion pour remercier les cabinets médicaux, les centres de soins ambulatoires et tous les médecins. Ils ont rendu ces résultats possibles grâce à leur transparence et à la transmission de leurs données à l'OFS et aux organisations de médecins.

\section{Références}

1 Premier relevé «Données structurelles des cabinets médicaux et centres ambulatoires» (MAS 2015): analyse de la participation et de la population, Office fédéral de la statistique (OFS), 20.3.2018; https://www.bfs.admin.ch/bfs/fr/home/statistiques/sante/enquetes/sdapaz.assetdetail.4842233.html

Pour les données du relevé MAS 2015 du tableau 2, il est $\quad 2$ Statistique des cabinets médicaux et des centres ambulatoires important de rappeler que les médecins exerçant dans _ (MAS), Office fédéral de la statistique (OFS), 10.4.2018; https://www.bfs.admin.ch/bfs/fr/home/statistiques/cataloguesbanques-donnees/publications.assetdetail.4924761.html 